PROCEEDINGS OF THE

AMERICAN MATHEMATICAL SOCIETY

Volume 137, Number 9, September 2009, Pages 3037-3044

S 0002-9939(09)09762-7

Article electronically published on March 24, 2009

\title{
ANALYTICITY OF COMPACT COMPLEMENTS OF COMPLETE KÄHLER MANIFOLDS
}

\author{
BOUDJEMÂA ANCHOUCHE
}

(Communicated by Mei-Chi Shaw)

\begin{abstract}
Let $X$ be a Stein manifold, $\operatorname{dim}_{\mathbb{C}} X \geq 2, K$ a compact subset of $X$, and $\Omega$ an open subset of $X$ containing $K$ such that $\Omega \backslash K$ is connected. Suppose that $\Omega \backslash K$ carries a complete Kähler metric of bounded bisectional curvature, and locally of finite volume near $K$. If $K$ admits a Stein neighborhood $V$, $V \subseteq \Omega$, such that $V \backslash K$ is connected and $H^{2}(V, \mathbb{R})=0$, then $K$ is a complex analytic subvariety of $X$, hence reduced to a finite number of points.
\end{abstract}

\section{INTRODUCTION}

In 4, H. Grauert constructed complete Kähler metrics on $X \backslash A$, where $X$ is a Stein manifold, $\operatorname{dim}_{\mathbb{C}} X \geq 2$, and $A$ is a closed analytic subvariety of $X$. In [7, T. Ohsawa proved that if $X$ is a complex manifold and $A$ is a $C^{1}$-submanifold of $X$ of real codimension 2 , such that $X \backslash A$ admits a complete Kähler metric, then $A$ is a complex submanifold of $X$. Later, K. Diederich and J. Fornaess, 3], considered the higher codimension case of Ohsawa's result. They proved that if $A$ is a closed real analytic subvariety of a complex manifold $X, \operatorname{dim}_{\mathbb{C}} X \geq 2$, of real codimension $\geq 3$, and if $X \backslash A$ is the support of a complete Kähler metric, then $A$ is complex analytic. In the same paper [3] they constructed, for each integer $k \geq 3$, a $C^{\infty}$-submanifold $A$ of the unit ball $\mathbb{B}^{n}$ in $\mathbb{C}^{n}$ of codimension $k$, which is not complex analytic, and for which $\mathbb{B}^{n} \backslash A$ carries a complete Kähler metric. Note that by Grauert's result, 4, the existence of a complete Kähler metric on a complex manifold $M$ does not imply the Steinness of $M$, for, it is enough to take $M=X \backslash A$, where $X$ is a Stein manifold, $\operatorname{dim}_{\mathbb{C}} X \geq 2$, and $A$ is a closed analytic subvariety of $X$ satisfying $\operatorname{codim}_{\mathbb{C}} A \geq 2$.

The aim of this paper is to give a sufficient condition for the analyticity of a compact subset of a Stein manifold of dimension $\geq 2$. More precisely, we prove the following:

Theorem 1.1. Let $X$ be a Stein manifold, $\operatorname{dim}_{\mathbb{C}} X \geq 2, K$ a compact subset of $X$, and $\Omega$ an open subset of $X$ containing $K$ such that $\Omega \backslash K$ is connected. Suppose that $\Omega \backslash K$ carries a complete Kähler metric $g$, of bounded bisectional curvature, and locally of finite volume near $K$; i.e., for every $x \in K$ and for every neighborhood $U$

Received by the editors October 10, 2007, and, in revised form, July 18, 2008, and November 10, 2008.

2000 Mathematics Subject Classification. Primary 32T05, 32Q15.

Key words and phrases. Analyticity of compact sets, Stein manifolds, complete Kähler metrics.

(C)2009 American Mathematical Society

Reverts to public domain 28 years from publication 
of $x$ such that $U$ is relatively compact in $\Omega$, we have

$$
\int_{U \backslash K} \omega_{g}^{n}<\infty,
$$

where $\omega_{g}$ is the $(1,1)$ form associated with the Kähler metric $g$. If $K$ admits a Stein neighborhood $V, V \subseteq \Omega$, such that $V \backslash K$ is connected and $H^{2}(V, \mathbb{R})=0$, then $K$ is a complex analytic subvariety of $X$, hence reduced to a finite number of points.

The proof of this result follows very closely the scheme developed by Mok and Zhong in [6]. We prove first that certain $d$-closed positive $(1,1)$ forms associated with the Kähler metric extend across some closed sets as $d$-closed positive $(1,1)$ currents. By comparing the potentials of the extended currents and by using the completeness of the metric, combined with Bombieri's lemma (see Lemma 4.1 below), we deduce the theorem.

\section{Extension of the Kähler $(1,1)$ FORM}

In this section we will prove that the $(1,1)$ form $\omega_{g}$, associated with the Kähler metric $g$, seen as a $d$-closed positive $(1,1)$ current, extends as a $d$-closed positive $(1,1)$ current across the set $K$. Let us first recall the following:

Lemma 2.1 (Yau's Maximum Principle, [8]). Let $(M, g)$ be a complete Riemannian manifold with Ricci curvature bounded from below, and let $v$ be a $C^{2}$-function which is bounded on $M$. Then for any $\varepsilon>0$, there exists a point $x_{\varepsilon} \in M$ such that

$$
\left|\nabla_{g} v\left(x_{\varepsilon}\right)\right| \leq \varepsilon, \Delta_{g} v\left(x_{\varepsilon}\right) \leq \varepsilon \text {, and } v\left(x_{\varepsilon}\right) \leq \inf v+\varepsilon,
$$

where $\nabla_{g}$ (resp. $\Delta_{g}$ ) is the gradient (resp. Laplacian) with respect to the metric $g$.

In what follows, the notation is that of Theorem 1.1. Since $X$ is a Stein manifold, there exists an embedding

$$
j: X \hookrightarrow \mathbb{C}^{N},
$$

for some positive integer $N$ ( $N$ can be taken to be $\left.2 \operatorname{dim}_{\mathbb{C}} X+1\right)$ such that $j(X)$ is a closed submanifold of $\mathbb{C}^{N}$. Let $\Omega_{0}$ be a relatively compact open subset of $\Omega$ such that $K \subset \Omega_{0}, \Omega_{0} \backslash K$ is connected and $\partial \Omega_{0}$ is smooth, and let $\mathbb{B}_{0}^{N}(R)$ be the Euclidean ball of $\mathbb{C}^{N}$ with center zero and radius $R$ such that $j\left(\Omega_{0}\right)$ is relatively compact in $\mathbb{B}_{0}^{N}(R)$.

Proposition 2.2. Let

$$
f=j_{\mid \Omega_{0} \backslash K}:\left(\Omega_{0} \backslash K, g\right) \hookrightarrow\left(\mathbb{B}_{0}^{N}(R), g_{\text {Berg }}\right),
$$

where $g_{\text {Berg }}$ is the Bergman metric on the Euclidean ball $\mathbb{B}_{0}^{N}(R)$. Then there exists a positive constant $C$ such that

$$
f^{*} g_{\text {Berg }} \leq C g \text { in } \Omega_{0} \backslash K .
$$

Proof. Let $u:=\operatorname{Tr}_{g}\left(f^{*} g_{\text {Berg }}\right)$. Proving the proposition is equivalent to proving that $u$ is bounded from above in $\Omega_{0} \backslash K$. To achieve that, we apply Yau's Maximum Principle (Lemma 2.1) to the function $v=\frac{1}{\sqrt{\chi u+1}}$, where $\chi$ is a cutoff function which will be defined below. Fix an open subset $\Omega_{1}$ of $\Omega$ such that $K \subset \Omega_{1} \Subset \Omega_{0}$. Since the metric $g_{\mid \Omega_{0} \backslash K}$ is not complete, and since Yau's result is Riemannian in nature, by perturbing the metric $g$ we will construct a complete Riemannian metric $\widetilde{g}$ in $\Omega_{0} \backslash K$, of bounded sectional curvature, hence of Ricci curvature bounded 
from below, satisfying $\widetilde{g}_{\mid \Omega_{1} \backslash K}=g_{\mid \Omega_{1} \backslash K}$. The construction goes as follows: by assumption, there exists a function $\rho \in C^{\infty}(\Omega)$ such that

$$
\Omega_{0}=\{x \in \Omega \mid \rho(x)<0\} \text { and } d \rho_{\mid \partial \Omega_{0}} \neq 0 .
$$

Choose a tubular neighborhood $T$ of $\partial \Omega_{0}$ such that $\bar{T} \cap \bar{\Omega}_{1}=\emptyset$, and let $\chi \in$ $C_{0}^{\infty}\left(\Omega_{0}\right), 0 \leq \chi \leq 1$, be such that $\chi_{\mid \Omega_{1}} \equiv 1$ and $\chi_{\mid T \cap\left(\Omega_{0} \backslash K\right)} \equiv 0$. Put

$$
\widetilde{g}:=\chi g+\frac{(1-\chi)}{\rho^{2}} g .
$$

Then $\widetilde{g}$ is a metric which satisfies all the properties mentioned above.

By Lemma 2.1 for each positive integer $n$, there exists a point $x_{n}$ in $\Omega_{0} \backslash K$ such that

$$
\left|\nabla_{\widetilde{g}} v\left(x_{n}\right)\right| \leq \frac{1}{n}, \Delta_{\widetilde{g}} v\left(x_{n}\right) \leq \frac{1}{n}, \text { and } v\left(x_{n}\right) \leq \inf v+\frac{1}{n},
$$

where $\nabla_{\widetilde{g}}$ (resp. $\Delta_{\tilde{g}}$ ) is the gradient (resp. Laplacian) with respect to the metric $\widetilde{g}$. Since $v$ achieves its maximum outside $\operatorname{supp}(\chi)$, there are two possibilities for the sequence $\left(x_{n}\right)_{n \in \mathbb{N}}$ : either $i$ ) there exists a subsequence $\left(x_{n_{j}}\right)_{j \in \mathbb{N}}$ converging to a point $x_{0} \in \Omega_{0} \backslash K$, or $\left.i i\right)$ all accumulation points of the sequence $\left(x_{n}\right)_{n \in \mathbb{N}}$ are in $K$. Suppose that case $i$ ) holds. Then $v$ has a minimum at $x_{0}$ with $\chi\left(x_{0}\right)>0$ (since $v$ has a maximum outside $\operatorname{supp}(\chi)$ ), and for any point $x \in \Omega_{0} \backslash K$, we have $v(x) \geq v\left(x_{0}\right)$, i.e.,

$$
\frac{1}{\sqrt{\chi(x) u(x)+1}} \geq \frac{1}{\sqrt{\chi\left(x_{0}\right) u\left(x_{0}\right)+1}} .
$$

From the definition of $\chi$ and the inequality 2.1 we deduce that

$$
u(x)=\chi(x) u(x) \leq \chi\left(x_{0}\right) u\left(x_{0}\right) \text { for all } x \in \Omega_{1} \backslash K .
$$

It is obvious that there exists a positive constant $c$ such that $u(x) \leq c$ for all $x \in \Omega_{0} \backslash \Omega_{1}$. Hence there exists a positive constant $C$ such that

$$
u(x) \leq C \text { for all } x \in \Omega_{0} \backslash K .
$$

In case $i i$ ), since the accumulation points of the sequence $\left(x_{n}\right)_{n \in \mathbb{N}}$ are in $K$, by ignoring at most a finite number of points of the sequence $\left(x_{n}\right)_{n \in \mathbb{N}}$ we can assume that $x_{n} \in \Omega_{1}$ for all $n$. Since $\widetilde{g}_{\mid \Omega_{1} \backslash K}=g_{\mid \Omega_{1} \backslash K}$, following the proof in 9 , we deduce that there exists a positive constant $C$ such that

$$
u(x) \leq C \text { for all } x \in \Omega_{1} \backslash K .
$$

The same argument as before implies the boundedness from above of $u$ in $\Omega_{0} \backslash K$. Hence the proposition follows.

Corollary 2.3. Under the assumptions of Theorem 1.1, the $(1,1)$ form $\omega_{g}$ extends to $\Omega$ as a positive $(1,1)$ current, denoted by $\widetilde{\omega}_{g}$.

Proof. The notation is as above. Consider the map

$$
f=j_{\mid \Omega_{0} \backslash K}:\left(\Omega_{0} \backslash K, \omega_{g}\right) \hookrightarrow\left(\mathbb{B}_{0}^{N}(R), \omega_{\text {Berg }}\right),
$$

where $\omega_{\text {Berg }}$ is the $(1,1)$ form associated with the Bergman metric $g_{\text {Berg }}$ on $\mathbb{B}_{0}^{N}(R)$. Then by Proposition 2.2 there exists a positive constant $c$ such that

$$
f^{*} \omega_{\text {Berg }} \leq c \omega_{g}
$$


Let $W$ be an open subset of $\mathbb{B}_{0}^{N}(R)$ such that $j\left(\Omega_{0}\right) \Subset W \Subset \mathbb{B}_{0}^{N}(R)$. Then there exists a positive constant $\widetilde{c}$ such that

$$
\omega_{\mathrm{ec}} \leq \widetilde{c} \omega_{\mathrm{Berg}} \text { in } W
$$

where $\omega_{\text {ec }}$ stands for the Kähler form associated with the Euclidean metric on $\mathbb{C}^{N}$. Let $x \in K$ and let $U$ be an open neighborhood of $x$ such that $U \subseteq \Omega_{1}$. Then, the inequalities (2.2) and (2.3) and the fact that $\widetilde{g}_{\mid \Omega_{1} \backslash K}=g_{\mid \Omega_{1} \backslash K}$ imply that

$$
\int_{U \backslash K} \omega_{g} \wedge\left(f^{*} \omega_{\mathrm{ec}}\right)^{n-1} \leq(c \widetilde{c})^{n-1} \int_{U \backslash K} \omega_{g}^{n}<\infty .
$$

We conclude that the $(1,1)$ form $\omega_{g}$ is of finite mass near $K$, hence extends (trivial extension) as a positive $(1,1)$ current, denoted by $\widetilde{\omega}_{g}$.

Proposition 2.4. Under the assumptions of Theorem 1.1, the $(1,1)$ form $\omega_{g}$ extends to $\Omega$ as a d-closed positive $(1,1)$ current, denoted by $\widetilde{\omega}_{g}$.

Proof. Since we have already proved that the trivial extension $\widetilde{\omega}_{g}$ of $\omega_{g}$ exists (Corollary 2.3), the proposition will follow if we prove that the extended positive $(1,1)$ current $\widetilde{\omega}_{g}$ is $d$-closed. By definition of the trivial extension, we have

$$
\widetilde{\omega}_{g}=\lim _{r \longrightarrow \infty} \rho_{r}(x) \omega_{g} \text { as currents, }
$$

where $\rho_{r}(x)$ is a cutoff function. Since the trivial extension of $\widetilde{\omega}_{g}$ does not depend on the choice of the cutoff function, we will prove that $\widetilde{\omega}_{g}$ is $d$-closed by an appropriate choice of $\rho_{r}(x)$. Let $x_{0}$ be a fixed point of $\Omega \backslash K$, and let

$$
\rho_{r}(x)=\left\{\begin{array}{l}
1 \text { if } x \in B_{g}\left(x_{0}, r\right) ; \\
-\frac{1}{3 r} d_{g}\left(x_{0}, x\right)+\frac{4}{3} \text { if } x \in B_{g}\left(x_{0}, 3 r\right) \backslash B_{g}\left(x_{0}, r\right) ; \\
0 \text { if } x \notin B_{g}\left(x_{0}, 4 r\right),
\end{array}\right.
$$

where $d_{g}\left(x_{0}, x\right)$ is the geodesic distance from $x_{0}$ to $x$, and $B_{g}\left(x_{0}, t\right)$ is the geodesic ball of center $x_{0}$ and radius $t$ in terms of the metric $g$. To prove that $\widetilde{\omega}_{g}$ is $d$-closed is equivalent to proving that

$$
d \widetilde{\omega}_{g}=\lim _{r \longrightarrow \infty} d \rho_{r}(x) \wedge \omega_{g}=0, \text { as currents. }
$$

For this it suffices to prove that

$$
\lim _{r \longrightarrow \infty} \int_{\Omega \backslash K}\left\|d \rho_{r}(x) \wedge \omega_{g} \wedge \psi\right\|=0,
$$

where $\psi$ is a $(2 n-3)$ smooth form, with compact support in $\Omega$. Following Mok and Zhong, [6], we can assume without loss of generality that $\psi=\psi_{1} \wedge\left(f^{*} \omega_{\mathrm{ec}}\right)^{n-2}$ and support $\psi_{1} \subset V$, where $V$ is a neighborhood of $K, V \subseteq \Omega_{0}$. As a consequence of the elementary inequality

$$
2 a b \leq r a^{2}+\frac{1}{r} b^{2}, r>0,
$$


we get

$$
\begin{aligned}
& 2 \int_{V \backslash K}\left\|\sqrt{-1} \bar{\partial} \rho_{r} \wedge \omega_{g} \wedge \psi_{1} \wedge\left(f^{*} \omega_{\mathrm{ec}}\right)^{n-2}\right\| \\
& \leq r \int_{V \backslash K}\left\|\omega_{g} \wedge \sqrt{-1} \partial \rho_{r} \wedge \bar{\partial} \rho_{r} \wedge\left(f^{*} \omega_{\mathrm{ec}}\right)^{n-2}\right\| \\
& \quad+\frac{1}{r} \int_{V \backslash K}\left\|\omega_{g} \wedge \sqrt{-1} \psi_{1} \wedge \bar{\psi}_{1} \wedge\left(f^{*} \omega_{\mathrm{ec}}\right)^{n-2}\right\| .
\end{aligned}
$$

But

$$
\sqrt{-1} \partial \rho_{r} \wedge \bar{\partial} \rho_{r} \leq \frac{1}{9 r^{2}} \omega_{g} \text { and } \sqrt{-1} \psi_{1} \wedge \bar{\psi}_{1} \leq c f^{*} \omega_{\mathrm{ec}}
$$

By combining the inequalities (2.4), (2.5), and (2.6) we obtain

$$
\begin{aligned}
2 \int_{V \backslash K}\left\|\sqrt{-1} \bar{\partial} \rho_{r} \wedge \omega_{g} \wedge \psi_{1} \wedge\left(f^{*} \omega_{\mathrm{ec}}\right)^{n-2}\right\| & \frac{1}{9 r} \int_{V \backslash K} \omega_{g}^{2} \wedge\left(f^{*} \omega_{\mathrm{ec}}\right)^{n-2} \\
& +\frac{c}{r} \int_{V \backslash K} \omega_{g} \wedge\left(f^{*} \omega_{\mathrm{ec}}\right)^{n-1} \\
& \leq \frac{C}{r} \int_{V \backslash K} \omega_{g}^{n} \leq \frac{C^{\prime}}{r},
\end{aligned}
$$

where the constant $C^{\prime}$ is independent of $r$. The result is obtained by taking the limit as $r$ goes to infinity. Hence the proposition follows.

\section{Extension of the tautological (1.1) FORM}

Let $(E, h)$ be a Hermitian vector bundle of rank $r$ over a complex manifold of dimension $n$. To $(E, h)$ is associated a Hermitian holomorphic line bundle $(L(E), \hat{h})$, on the projectivisation $\mathbb{P}(E)$ of $E$, called the tautological line bundle. The line bundle $L(E)$ is the subbundle of $\pi^{*} E$ whose fiber $L(E)_{[\xi]}$ at the point $[\xi] \in \mathbb{P}(E)$ is the line in $E_{\pi([\xi])}$ represented by $[\xi]$, where $\pi: \mathbb{P}(E) \longrightarrow M$ is the projection onto $M$. The map $\pi$ induces a biholomorphism outside of the trivial sections of $L(E)$ and $E$, i.e., $L(E) \backslash \mathbb{P}(E) \cong E \backslash M$. Hence the metric $h$ on $E$ induces a metric $\hat{h}$ on $L(E)$.

In what follows, we calculate the curvature of the metric $\hat{h}$ in terms of that of $h$. Let $x_{0} \in M,\left(U, z_{1}, \ldots, z_{n}\right)$ be a chart centred at $x_{0}$, i.e., $z_{i}\left(x_{0}\right)=0$, and let $\left(e_{1}(z), \ldots, e_{r}(z)\right)$ be a special holomorphic basis of $E$, i.e., $h\left(e_{i}, e_{j}\right)(0)=\delta_{i j}$ and $d h\left(e_{i}, e_{j}\right)(0)=0$. Let $\eta_{0}$ be a unitary vector in the fiber $E_{x_{0}}$ and $\left[\eta_{0}\right]=v_{0} \in P(E)$. By applying a unitary transformation to the vector $\left(e_{1}(z), \ldots, e_{r}(z)\right)$ we can assume that $\eta_{0}=e_{r}(0)$. For $\eta=\sum_{j=1}^{r} \eta_{j} e_{j} \in E$ we take $\left(z_{1}, \ldots, z_{n}, \eta_{1}, \ldots, \eta_{r}\right)$ as local holomorphic coordinates on $E$, and since $L(E)$ coincides with $E$ outside of their trivial sections, $\left(z_{1}, \ldots, z_{n}, \eta_{1}, \ldots, \eta_{r}\right)$ may be used as a local holomorphic coordinate for $L(E)$ outside of the trivial section. At the point $\eta_{0}$ we have $\eta_{1}=\ldots=\eta_{r-1}=$ $0, \eta_{r}=1$. Put $w_{\alpha}=\eta_{\alpha} / \eta_{r}, \alpha=1, \ldots, r-1$. Then $\left(z_{1}, \ldots, z_{n}, w_{1}, \ldots, w_{r-1}\right)$ is a system of holomorphic coordinates in an open neighborhood $\widetilde{U}_{\nu_{0}}$ of the point 
$v_{0}=\left[\eta_{0}\right] \in P(E)$, and the map

$$
\begin{aligned}
& s: \quad \mathbb{P}(E) \supset \widetilde{U}_{v_{0}} \quad \longrightarrow \quad L(E) \\
& \left(z_{1}, \ldots, z_{n}, w_{1}, \ldots, w_{r-1}\right) \longmapsto\left(z_{1}, \ldots, z_{n}, w_{1}, \ldots, w_{r-1}, 1\right)
\end{aligned}
$$

is a special holomorphic basis of $L(E)$ at the point $v_{0}$ denoted by $\hat{e}$. Using (3.1), it can be proved that the curvature $\Theta(L(E), \hat{h})$ of the metric $\widehat{h}$ on $L(E)$, induced from the metric $h$ on $E$, is given by 1

$$
\Theta(L(E), \hat{h})=-\frac{\sqrt{-1}}{2 \pi} \partial \bar{\partial} \log \left[\sum_{\alpha, \beta=1}^{r-1} h_{\alpha \bar{\beta}}(z) w_{\alpha} \bar{w}_{\beta}+2 \operatorname{Re} \sum_{\alpha=1}^{r-1} h_{\alpha \bar{r}}(z) w_{\alpha}+h_{r \bar{r}}(z)\right] .
$$

If $(M, g)$ is a Kähler manifold, of bounded bisectional curvature, then there exists a positive constant $k$ such that

$$
\mathcal{T}_{g, k}=-\Theta(L(T M), \widehat{g})+k \pi^{*} \omega_{g}
$$

is a $(1,1)$ positive form, where $\Theta(L(T M), \widehat{g})$ is the curvature of the tautological line bundle associated to the holomorphic tangent bundle $(T M, g)$ of the manifold $M, \omega_{g}$ is the $(1,1)$ form associated to the metric $g$ and $\pi: \mathbb{P} T M \longrightarrow M$ is the projection onto $M$. Hence $\left(\mathbb{P} T M, \mathcal{T}_{g, k}\right)$ is a Kähler manifold.

In what follows, we take $M=\Omega \backslash K$ and choose a constant $k$ such that the "tautological" $(1,1)$ form $\mathcal{T}_{g, k}$ is a Kähler form defined on $\mathbb{P} T(\Omega \backslash K)$. Since $X$ is Stein, the tangent bundle $T X$ is trivial, and therefore $\mathbb{P} T(\Omega \backslash K) \cong(\Omega \backslash K) \times \mathbb{P}^{n-1}$.

Proposition 3.1. Under the assumptions of Theorem 1.1, the tautological $(1,1)$ form $\mathcal{T}_{g, k}$ extends to $\mathbb{P} T \Omega$ as a d-closed positive $(1,1)$ current, denoted by $\widetilde{\mathcal{T}}_{g, k}$.

Proof. Fix $[\xi] \in \mathbb{P}^{n-1}$, and consider the following $(1,1)$ form $\left(\mathcal{T}_{g, k}\right)_{\mid(\Omega \backslash K) \times[\xi]}$. From the definition of $\mathcal{T}_{g, k}$, we deduce that there exists a constant $C$ such that

$$
\left(\mathcal{T}_{g, k}\right)_{\mid(\Omega \backslash K) \times[\xi]} \leq C\left(\pi^{*} \omega_{g}\right)_{\mid(\Omega \backslash K) \times[\xi]} \text { as }(1,1) \text { forms. }
$$

Since $\left(\pi^{*} \omega_{g}\right)_{\mid(\Omega \backslash K) \times[\xi]}$ is locally of finite volume near $K$, by the same arguments as in section 2, we deduce that $\left(\mathcal{T}_{g, k}\right)_{\mid(\Omega \backslash K) \times[\xi]}$ extends to $\Omega \times[\xi]$ as a $d$-closed positive current. Since $[\xi] \in \mathbb{P}^{n-1}$ is arbitrary, we deduce that $\mathcal{T}_{g, k}$, seen as a $d$-closed positive $(1,1)$ form, extends to $\mathbb{P} T \Omega$ as a $d$-closed positive $(1,1)$ current, denoted by $\widetilde{\mathcal{T}}_{g, k}$. Hence the proposition follows.

\section{Proof of the Main Theorem}

Recall first the following very well-known result of Bombieri.

Lemma 4.1 (E. Bombieri, [1, 2]). Let $M$ be a complex manifold, let @ be a plurisubharmonic function on $M$, and let

$$
A=\left\{z \in M \mid e^{-\varrho} \text { is not integrable in a neighborhood of } z\right\} .
$$

Then $A$ is an analytic subset of $M$.

Since analyticity is a local property, to prove that $K$ is complex analytic it is enough to prove it locally in a neighborhood of each point $x$ in $K$. By comparing the potentials of the $d$-closed positive $(1,1)$ currents $\widetilde{\omega}_{g}$ and $\widetilde{\mathcal{T}}_{g, k}$, we construct a plurisubharmonic function in a neighborhood of $x \in K$. The completeness of the

\footnotetext{
${ }^{1}$ For more details, see 5, Chapter III.
} 
metric reduces the problem of the analyticity of $K$ in a neighborhood of $x \in K$ to a straightforward application of Bombieri's lemma.

Proposition 4.2. Let $x_{0} \in K$ and let $\varrho$ be a potential for the $d$-closed positive $(1,1)$ current $\widetilde{\omega}_{g}$ in some neighborhood $V \subset \Omega$ of $x_{0}$. Then there exists a positive constant $\gamma$ (independent of the point $x_{0}$ ) such that for every neighborhood $V^{\prime} \subseteq V$ of $x_{0}$, we have

$$
\int_{V^{\prime}} e^{-\gamma \varrho(x)} d \lambda(x)=\infty
$$

where $d \lambda$ is the Lebesgue measure. As a consequence, $K$ is an analytic subvariety of $X$, hence reduced to a finite number of points.

Proof. Let $x_{0} \in K$, and let $\left(z_{1}, \ldots, z_{n}\right)$ be global holomorphic coordinates centred at $x_{0}$, i.e., $z_{j}\left(x_{0}\right)=0$ for $j=1, \ldots, n$. Consider the point $P=\left(z_{1}, \ldots, z_{n},\left[\partial / \partial z_{n}\right]\right)$ of $\mathbb{P} T \Omega \cong \Omega \times \mathbb{P}^{n-1}$, and let $v=\sum_{i=1}^{n} v_{i} \partial / \partial z_{i}$ be a holomorphic vector field in $\Omega$, where $v_{n} \neq 0$. Then $\left(\left(z_{i}\right)_{1 \leq i \leq n},\left(w_{\alpha}\right)_{1 \leq \alpha \leq n-1}\right)$ constitutes a system of holomorphic coordinates in a neighborhood $U^{\prime}=\Omega \times U$ of $P$, where $w_{\alpha}=v_{\alpha} / v_{n}, \alpha=1, \ldots, n-1$, and $U$ is a polydisc, in the coordinates $\left(w_{\alpha}\right)$, which is a neighborhood of $\left[\partial / \partial z_{n}\right]$ in $\mathbb{P}^{n-1}$. Recall that we have proved that the $(1,1)$ form $\mathcal{T}_{g, k}$ (resp. $\left.\omega_{g}\right)$ defined in $\mathbb{P} T(\Omega \backslash K) \cong(\Omega \backslash K) \times \mathbb{P}^{n-1}$ (resp. in $\Omega \backslash K$ ) extends to $\mathbb{P} T \Omega$ (resp. $\Omega$ ) as a positive $d$-closed $(1,1)$ current denoted by $\widetilde{\mathcal{T}}_{g, k}$ (resp. $\widetilde{\omega}_{g}$ ). By assumption, there exists a Stein subset $V$ of $\Omega$ such that $K \subset V, V \backslash K$ is connected, $H^{2}(V ; \mathbb{R})=$ 0 , and $H^{2}(V \times U ; \mathbb{R})=0$. Therefore there exists in $U^{\prime}=V \times U($ resp. $V)$ a plurisubharmonic function $\psi$ (resp. @) such that

$$
\widetilde{\mathcal{T}}_{g, k}=\sqrt{-1} \partial \bar{\partial} \psi
$$

resp.

$$
\widetilde{\omega}_{g}=\sqrt{-1} \partial \bar{\partial} \varrho .
$$

From (3.2), (3.3), (4.1), and (4.2), we deduce that

$$
\sqrt{-1} \partial \bar{\partial}\left(\frac{1}{2 \pi} \log \left[\sum_{i, j=1}^{n-1} g_{i \bar{j}}(z) w_{i} \bar{w}_{j}+2 \operatorname{Re} \sum_{i=1}^{n-1} g_{i \bar{n}}(z) w_{i}+g_{n \bar{n}}(z)\right]+k \pi^{*} \varrho-\psi\right)=0 \text {, }
$$

in $(V \backslash K) \times U$. Hence

$$
\frac{1}{2 \pi} \log \left[\sum_{i, j=1}^{n-1} g_{i \bar{j}}(z) w_{i} \bar{w}_{j}+2 \operatorname{Re} \sum_{i=1}^{n-1} g_{i \bar{n}}(z) w_{i}+g_{n \bar{n}}(z)\right]+k \pi^{*} \varrho=\psi+h,
$$

where $h$ is a pluriharmonic function on $(V \backslash K) \times U$.

Since $V \backslash K$ is connected, every holomorphic function on $V \backslash K$ extends to a holomorphic function on $V$. Hence $\mathcal{H}((V \backslash K) \times U)=\mathcal{H}(V \backslash K) \times U=V \times U$, where $\mathcal{H}((V \backslash K) \times U)$ (resp. $\mathcal{H}(V \backslash K)$ ) means the envelope of holomorphy of $(V \backslash K) \times U$ (resp. $V \backslash K)$. We deduce by standard arguments that $h$ extends to a plurisubharmonic function $\widetilde{h}$ on $V \times U$. After shrinking $V$ (resp. $U^{\prime}$ ) if necessary, we can assume that $\varrho($ resp. $\psi, \widetilde{h})$ is bounded from above in $V$ (resp. $\left.U^{\prime}\right)$. If we restrict the equation (4.4) to the unit disc $\Delta$ defined by

$$
\Delta=\left\{\left(z_{i}, w_{\alpha}\right) \in V \times U \mid z_{1}=\ldots=z_{n-1}=w_{1}=\ldots=w_{n-1}=0\right\},
$$


we get

$$
\frac{1}{2 \pi} \log \left[g_{n \bar{n}}\left(0, z_{n}\right)\right]=\psi\left(0, z_{n}, 0\right)+h\left(0, z_{n}, 0\right)-k \varrho\left(0, z_{n}\right) .
$$

The completeness of the metric $g$ in a neighborhood of $x_{0} \in K$ implies that

$$
\int_{0}^{\varepsilon} \sqrt{g_{n \bar{n}}\left(0, r e^{i \theta}\right)} d r=\infty,
$$

where $\varepsilon>0$ is small enough. Combining equations (4.5) and (4.6) we can show, as was done by Mok and Zhong in [6], that there exists a positive constant $\gamma$ which is independent of $x_{0} \in K$ such that for every neighborhood $\Upsilon$ of $x_{0}$, we have

$$
\int_{\Upsilon} e^{-\gamma \varrho(z)} d \lambda(z)=\infty .
$$

In other words, $e^{-\gamma \varrho}$ is not locally integrable in a neighborhood of $x_{0} \in K$. But $x_{0}$ is arbitrary, and then by Lemma 4.1, $K$ is a complex analytic subvariety of $V$ and hence reduced to a finite number of points.

\section{ACKNOWLEDGEMENTS}

The author is grateful to the referee for his careful reading of the paper and for his comments and suggestions which helped to improve the presentation and the content of the paper We are grateful also to Professor J. P. Demailly for his comments on some questions related to this paper.

\section{REFERENCES}

[1] E. Bombieri, Algebraic values of meromorphic maps. Invent. Math 10 (1970), 267-287. MR0306201 (46:5328)

[2] E. Bombieri, Addendum to my paper: "Algebraic values of meromorphic maps". Invent. Math 11 (1970), 163-166. MR0322203 (48:565)

[3] K. Diederich and J. Fornaess, Thin complements of complete Kähler domains. Math. Annalen 259 (1982), 331-341. MR0661201 (84a:32017)

[4] H. Grauert, Charackterisierung der Holomorphiegebiete durch die vollständige Kählersche Metrik. Math. Ann. 131 (1956), 38-75. MR0077651(17:1072a)

[5] S. Kobayashi, Differential geometry of complex vector bundles. Publications of the Mathematical Society of Japan, no. 15, Princeton Univ. Press., Princeton, NJ; Iwanami Shoten Publishers, Tokyo, 1987. MR909698 (89e:53100)

[6] N. Mok and J. Q. Zhong, Compactifying complete Kähler-Einstein manifolds of finite topological type and bounded curvature. Annals of Mathematics (2) 129 (1989), 427-470. MR0997309 (90d:32045)

[7] T. Ohsawa, Analyticity of complements of complete Kähler domains. Proc. Japan Acad. Ser. A Math. Sci. 56 (1980), 484-487. MR0605768 (82j:32025)

[8] S. T. Yau, Harmonic functions on complete Riemannian manifolds, Comm. Pure Appl. Math. 28 (1975), 201-228. MR0431040 (55:4042)

[9] S. T. Yau, A general Schwarz lemma for Kähler manifolds. Amer. J. Math. 100 (1978), 197-203. MR0486659 (58:6370)

Department of Mathematics and Statistics, Sultan Qaboos University, Muscat, Oman

E-mail address: anchouch@squ.edu.om

\footnotetext{
${ }^{2}$ The paper was originally written in the context of bounded domains of holomorphy. The referee suggested we extend it to the general case of domains in Stein manifolds and kindly drew our attention to reference 8 in order to achieve that. Proposition 2.2 is due to him.
} 